gations: chest $x$-ray normal; Hb $55 \%$ W.B.C. 7,100 with normal diffierential; iron deficiency on the blood film; E.S.R. $138 \mathrm{~mm}$. in one hour; Paul-Bunnell test negative. Blood culture revealed Haemophilus influenzae type B sensitive to all the usual antibiotics except sulphonamide. This child was treated with ampicillin and made a good recovery.

My point in recording this case history is to emphasize that septicaemia due to Haemophilus influenzae may not be as rare as we had thought and one can easily envisage such a child as I have mentioned developing osteomyelitis due to this organism.-I am, etc.,

Southampton Children's Hospital,

J. L. Greaves.

Southampton, Hants.

\section{Monoamine Oxidase Inhibitors and Anaesthesia}

SIR,-The risks involved in anaesthetizing patients receiving monoamine oxidase inhibitors have been largely eliminated in the West Dorset Group of Hospitals by the adoption of the following routine for admission of cases from the surgical waiting-list.

(1) Every patient is instructed to bring with him any pills, medicines, etc., he is currently receiving.

(2) The patient's general practitioner is sent a form several days before the day of admission informing him of the proposed admission. This form is prestamped and returnable, and contains the following questionary:

"What drugs is the patient currently receiving? If the patient has received steroids during the past two years or monoamine oxidase inhibitors during the past two weeks please give details."

This procedure has been in operation for nearly two years and has proved to be very valuable, thanks to the co-operation of the general practitioners in the area.-I am, etc.,

Weymouth and District Hospital J. W. WARRICK.

\section{Idiopathic Facial Palsy}

SIR,-Mr. John Groves (24 February, p. 508) has performed a valuable service in drawing attention to the possibility that lack of properly matched controls vitiates the conclusion of Dr. D. Taverner and others (9 December 1967, p. 581) that A.C.T.H. is of proved benefit in the treatment of Bell' palsy. They say that they are convinced of its efficacy, but the fallibility of personal conviction or clinical impression is too well known to warrant further comment. Moreover, in that part of their previous trial where properly matched controls were used no significant difference between the treated and the untreated groups was shown.

The observation of $\mathrm{Mr}$. Groves, that as one sees cases in hospital series earlier in the course of Bell's palsy so does the denervation rate lessen, is confirmed by the following figures in a series of 128 cases of complete unilateral Bell's palsy referred for electrodiagnostic testing. These cases were seen on the first occasion at any time up to 28 days from the onset of the condition, and the denervation rate (the criteria for denervation being those described by Taverner et al. ${ }^{1}$ ) was $40 \%$. If out of this series those seen up to 21 days are studied then the denervation rate drops to $33 \%$. In cases up to 14 days the denervation rate is $29 \%$, up to 7 days $20 \%$, and if those seen only up to 5 days are selected the rate is $13 \%$.

None of those patients had any treatment designed to prevent denervation, and this denervation rate of $13 \%$ is the same as in Taverner's five-day series, in which those patients predicted to be in danger of denervation had been treated with A.C.T.H. Thus it becomes clear that a five-day treated series can be compared with a concurrent five-day untreated series only to give a valid comparison, whereas Taverner and his colleague have compared a five-day treated series retrospectively with a 14-day untreated, thus giving a built-in bias of considerable magnitude in favour of the method of treatment. This method of comparison amply illustrates the dictum quoted by Bradford Hill" "The assessment of therapeutic activity by the use of retrospective controls is an inherently fallacious method."

So far the criticisms and comments of $\mathrm{Mr}$. Groves have remained unanswered-perhaps because they are unanswerable.-I am, etc. London W.1.

E. D. R. Campbell.

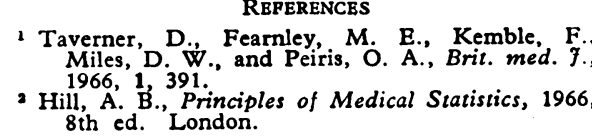

\section{Zinc Sulphate and Bedsores}

SIR,-Acceleration of the healing time of open wounds in a small group of young men who were given zinc sulphate $220 \mathrm{mg}$. three times daily by mouth has been reported, ${ }^{1}$ with no undesirable side-effects. The same treatment was given to six elderly people who had kedsores at the time of admission to geriatric unit.

Case 1.-80-year-old man with a bedsore on the right heel, $6.5 \mathrm{~cm}$. by $6 \mathrm{~cm}$. by $5 \mathrm{~cm}$.; full thickness loss of skin; present at least on month; no sign of healing. Zine sulp aate 220 m3. given three times daily for 33 days was associated with complete healing and new epithelium.

Case 2.-73-year-old man with diabetes who had a perforating ulcer on sole of right foot, $5 \mathrm{~cm}$. by $2 \mathrm{~cm}$.; full thickness loss of skin, and present " for a long time." Zinc sulphate was given three times daily $f=r 27$ days, after which partial healing was observed but patient insisted on going home. Fifty days later the patient was seen again. Treatment had probably been discontinued, but there remained only three "islands" of broken skin, $1 \mathrm{~cm} ., 1 \mathrm{~cm}$., and $0.5 \mathrm{~cm}$. in diameter.

Case 3.-70-year-old man with a bcdsore on his right heal, $6 \mathrm{~cm}$. by $6 \mathrm{~cm}$. by $5 \mathrm{~cm}$.; full thickness loss of skin and involvement of underlying tissues; present for at least four months. Zinc sulphate three times daily produced no effect for one month, after which healing began and became complete in 106 days.

Case 4.-70-year-old woman with bedsore on her right heel, $5 \mathrm{~cm}$. by $4 \mathrm{~cm}$., full thicknes loss of skin, and present for at least three months. Zinc sulphate was given three times dailv for 66 days, when complete healing was noted.

Case 5.-81-year-old woman with a superficial ulcer over the sacral region and bedsores on both heels, with full thickress loss of skin at the left heel. Zinc sulphate was given three times daily for 17 days, when both heels were noted to be healed.

Case 6.-84-year-old woman developed a deep sacral sore $8 \mathrm{~cm}$. by $6 \mathrm{~cm}$. with a track at least
$0.5 \mathrm{~cm}$. diameter into underlying tissue during treatment for a perforated gall bladder. The ulcer showed no sign of healing after 12 months, during which time energetic treatment included two surgical toilet operations. Zinc sulphate was given twice daily with no effect for about one month, after which healing was noted, and at the moment there is residual superficial loss of skin $2 \mathrm{~cm}$. by $0.5 \mathrm{~cm}$.

Pories et al." indicated that "zinc is the metal moiety in a number of essential enzymes," and that "zinc is preferentially concentrated in healing tissues with a peak activity in the first days after injury." Zinc sulphate was given in the above cases in addition to standard treatment for bedsores, and control cases were not included. Experienced members of the nursing staff thought that healing was promoted, the final stage of healing was not delayed compared with previous treatment regimens, and " new skin" appeared over the ulcer.

No ill effects have been seen in the six cases, and, though the initial results are encouraging, further evaluation of the effectiveness of zinc sulphate, the minimum dose required, and the mode of action is required. It is hoped that the above cases will be reported in more detail elsewhere.-I am, etc.,

Stracathro Hospital,

Cyril Cohen.

Brechin, Angus.

REFERENCB

$\underset{\text { Pories, W. J., Henzel, J. H., Rob, C. G., and }}{\text { He, }}$

\section{Paracervical Block with Bupivacaine}

SIR,-I have read with interest $\mathrm{Mr}$. D. H Gudgeon's article (18 May, p. 403).

I have just completed a series of 100 cases of paracervical block with bupivacaine $0.5 \%$ with adrenaline 1:200,000. My initial experience with $0.25 \%$ was the same as $\mathrm{Mr}$. Gudgeon's in that the duration of analgesia was about three hours. I did not feel that this was sufficiently long, and have found that the higher dosage gives a much improved duration of effect. We have, however, noted the occurrence of severe foetal bradycardia on a few occasions, and the occurrence of this complication has never been satisfactorily explained, but from the work of other authors adrenaline does not appear to be implicated. I feel that if Mr. Gudgeon's series had been rather longer foetal bradycardia might well have been observed.

It is hoped that our results will shortly be published.-I am, etc.,

$$
\begin{aligned}
& \text { Edgware General Hospital, } \\
& \text { Middlesex. }
\end{aligned}
$$

\section{Cirrhosis of the Liver}

SIR,-I was interested to read the article on cirrhosis of the liver by Dr. A. E. Read (17 February, p. 427), as it concisely and lucidly presented the generally accepted British views on hepatic cirrhosis. Certain differences stand out in marked contrast with the disease seen in India, where it is no less common. They are as follows.

Alcohol in the aetiology of disease can be totally ruled out. One hardly ever comes across a cirrhotic who has ever consumed alcohol. Cirrhosis in adults is also far more commo in Hindu males who are traditionally vegetarian. They come from a poorer sncin- 